\title{
BANDWIDTH ENABLED FLIGHT OPERATIONS: EXAMINING THE POSSIBILITIES
}

\author{
Greg Pisanich and Fritz Renema \\ QSS Group Inc., NASA Ames Research Center, Moffett Field, CA
}

\begin{abstract}
The Bandwidth Enabled Flight Operations project is a research effort at the NASA Ames Research Center to investigate the use of satellite communications to improve aviation safety and capacity. This project is a follow on to the AeroSAPIENT Project, which dernonstrated methods for transmitting high bandwidth data in various configurations. For this research, we set a goal to nominally use only 10 percent of the available bandwidth demonstrated by AeroSAPIENT or projected by neir-term technology advances.
\end{abstract}

This paper describes the results of our research, including available satellite bandwidth, commercial and research efforts to provide these services, and some of the limiting factors inherent with this communications medium. It also describes our investigation into the needs of the stakeholders (Airlines, Pilots, Cabin Crews, ATC, Maintenance, etc). The paper also describes our development of low-cost networked flight deck and airline operations center simulations that were used to demonstrate two application areas: Providing real time weather information to the commercial flight deck, and enhanced crew monitoring and control for airline operations cente:s.

\section{Introduction}

The Bandwidth Enabled Flight Operations (BEFO) project stemmed from the results of the AeroSAPIENT Project (Aeronautical Satellite Assisted Process for Information Exchange through Network Technologies). AeroSAPIENT [1] was based at the NASA Glenn Research Center (Ohio), and included collaboration with NASA Ames (CA), NASA Dryden (CA), and the Tinkt $r$ Air Force Base in Oklahoma.
The AeroSAPIENT project demonstrated technology that was able to support a high bandwidth computer network connection between a flying aircraft and a ground station via a satellite. A DC-8 research aircraft was used for the flight tests, through which in-flight network communications technology was used to enact several simultaneous applications at a high bandwidth data rate.

Based on these results, the Bandwidth Enabled Flight Operations (BEFO) project was initiated within the Computational Sciences Division at the NASA Ames Research Center. The high level goal of the project was to investigate new ways in which this technology could be applied to improve airline safety and capacity (the ability to move more aircraft and passengers through the airspace system). Our initial development direction and goals were to:

- Understand the current capacity and limitations of air-ground bandwidth within the commercial aviation industry.

- Understand what applications potential users (Airlines, Pilots, Cabin Crews, ATC, Maintenance) envisioned to use with increased bandwidth.

- Develop a prototype cockpit environment in which new concepts could be easily evaluated.

- $\quad$ Collaborate with other groups working on technologies that could be easily integrated into this environment.

The work described in this paper was performed between July 2001 and February 2002. 


\section{Aviation Bandwidth Issues}

\section{Transmission Speed and Capacity}

Our initial research efforts were to understand the capabilities of current aviation networking technology. Two examples of current aviation communication networks include CPDLC [2] and the FAA Capstone Project [3]. CPDLC (Controller Pilot Datalink Communications) is an effort to support both voice and data transmissions to and from the cockpit, with non-critical information exchanges being sent over the data line. This system operates at $2.4 \mathrm{Kbps}$ data and $9.6 \mathrm{Kbps}$ voice. The FAA Capstone Program installed avionics and data link communications suites in aircraft serving Alaska to test new technologies. This system operated at $19.2 \mathrm{Kbps}$ from and 3.6 Kbps to the aircraft.

Several higher bandwidth projects were being proposed at the time by Boeing, Tenzing \& Hughes Global Services, AirTV, and the Airshow network. These indicated that bandwidth ranges from $1.4 \mathrm{Kbps}$ to $1.5 \mathrm{Mbps}$ could be possible from the aircraft to the ground. They also indicated possible data rates from $144 \mathrm{Kbps}$ to $8 \mathrm{Mbps}$ from the ground to the aircraft. The high bandwidth capabilities demonstrated during the AeroSAPIENT research project indicated that data rates of $256 \mathrm{Kbps}$ from the plane and $2.18 \mathrm{Mbps}$ to the airplane were feasible [1].

An example of bandwidth usage is useful to help illustrate the amount necessary to support various applications of interest. Here are some typical "real-life" examples:

- Video/audio streaming server: $80 \mathrm{Kbps}$

- Voice over IP: 10Kbps

- $\quad$ RTSS streaming video from the Half Moon Bay airport (video and environmental conditions such as temperature and due point): $720 \mathrm{Kbps}$

Using these values, three video streams could saturate the entire data stream sent from the aircraft based on the AeroSAPIENT results.

\section{Other Factors}

There are other network related issues involved that could affect multiple-aircraft operations. These include limitations of the satellite technology. For example, transponders, which are microwave repeaters carried by communications satellites, are a limited resource. The AeroSAPIENT project used two separate transponders-one to transmit from the aircraft, and one to receive. In regards to operations with many aircraft at the same time, scalability will depend on the design and implementation of the total system, which will be a determining factor that impacts how much bandwidth will be available to each airplane.

Other factors involve communications frequency choice, ambient weather conditions (rain and fog can hamper operations-some frequencies being more susceptible than others), and the selection of spread spectrum or narrow beam transmission. Transmission power is another factor.

While these issues are important, they were not a major focus of our project and are beyond the scope of this paper.

\section{Bandwidth Limitations and Assumptions}

For this project, we were asked to work under a limitation of using a nominal ten percent of the total bandwidth (send and receive) made available by the AeroSAPIENT project. In emergency situations the amount could rise to $100 \%$. The resulting values of $25.6 \mathrm{Kbps}$ from and $218 \mathrm{Kbps}$ to the aircraft were established for several reasons.

First, our NASA Task Requestor foresaw that airlines would want to use the bandwidth to support profit-making opportunities and to improve service. Our future discussions with airline representatives supported this prediction.

We also initially thought that bandwidth needs might vary during different phases of flight just as pilot workload does. For example, a higher amount of bandwidth might be used during takeoff and climb, less in cruise, and more during descent and landing. Although most of the applications we identified in our research would be used during cruise, we still feel that these phase-of-flight 
differences in bandwidth usage w 11 bear out. These might be supported by some transmission technology other than the current satellite systems (for example, the transmission of high bandwidth data from terrestrial sites aligned with airport approach paths).

We also came to the conclusion early in the research that an airline or data provider could "multicast" much of the data needed by many aircraft. A system on board the aircraft could tune to the specific data feed when needed. Data could also be collected and stored over time by an aircraft traveling in flight and used at the point it was required.

We assumed that compression algorithms would be available to improve the transmission of this data. Where algorithms were available, we used them (for example, video compression algorithms), but we did not develcp our own.

\section{Researching Stakeholder Needs}

A major goal of this project was to better understand who would use this new capability and how it would be used. We spoke with over 50 individuals and groups within the aviation industry and airspace system. These included Airline pilots, cabin crew, operations, and maintenance personnel; ATC (Center, TRACON and Tower); Weather Providers and Flight Service personnel; and researchers from NASA Ames anc Glenn.

In the follow paragraphs we have summarized our identification of "needs", from the perspective of each of these groups:

\section{Pilots}

The pilots we interviewed were primarily interested in getting additional "bis picture" data which would allow them to see further ahead and around them in order to make better decisions during the flight.

For example, on board weather radar can often yield a false picture of what is actually happening due to a storm cell which is close to the airplane blocking information about storm cells located further along, which may be far worse. They also felt that this information would enable better planning which would increase fuel efficiency by allowing pilots to re-route their course, if necessary, near the beginning of their flight, thereby allowing a more direct course.

Pilots were also interested in using this system to enable better collaboration. An example might be that turbulence reports from other aircraft and the routes and altitudes they used to avoid turbulence could be shared more easily and quickly. Both pilots and cabin crew saw turbulence reporting as an important application, to include passenger comfort, safety, and a liability point of view.

Pilots were also interested in gaining access to better maintenance reporting. They wanted to be able to speak directly with maintenance personnel, which would allow subtle issues or problems with an aircraft to be discussed in real time rather than through a limited number of fault codes.

\section{Cabin Crew}

The cabin crew also wanted direct access to warnings of reported or possible turbulence without having to get the data from the pilots. Advanced warnings would allow them to plan serving operations around rough weather, short term warnings would allow them to lock down serving carts several seconds before turbulence showed up.

They also wanted the ability to report squawks (problems with the plane such as a seat that will not recline) directly to maintenance, and access to customer data (which connecting gate does the person in seat $14 \mathrm{~F}$ need, once we arrive at Chicago?).

\section{Air Traffic Control: Center, TRACON, Tower}

ATC was satisfied with the information that they had for the most part, but wanted more accurate position, speed, and intent data.

They also wanted a way of automatically verifying that a pilot has reviewed the ATIS information (which gives conditions and airport notices) for the airport of intended landing. Currently, if a pilot does not report having received the information, the air traffic controller must ask, and then either spend time informing the pilot of the 
latest information or requesting that the pilot tune to another frequency and listen to it themselves.

\section{Flight Service Stations/Weather Providers}

Both saw the capability of installing weather sensors onboard each commercial aircraft would have a tremendous impact on the development of weather models, especially for long flights overseas. These sensors could be programmed to record and download data at specific millibar levels. Currently, weather balloons are still the primary method of sensing this data from the environment.

\section{Airline Industry}

In talking with industry representatives, our thoughts that airline management needs to see a financial incentive in order to implement this technology were verified. Their first target involved revenue-enhancing applications, such as email or web surfing at a premium, for their passengers. Maintenance and flight operation enhancements were next, followed by cockpit enhancements. They also saw the value of collecting weather data with their aircraft, both for increasing safety and as a possible revenue stream.

At the time that we talked with them (August, 2001), at least one airline was in the process of developing an infrastructure to support broadband into their fleet. They were looking at how transponders, ground support and phased array antennas would be used to support the above applications. They also were in discussions with both Boeing and Airbus on how this hardware would be integrated. Several other airlines were interested in their system.Benefit Areas

Based on this research, we identified three major areas where we felt we should focus our development to provide the greatest benefits.

\section{Providing Relevant Information}

The first area was to provide relevant information to the user. This would not be raw data, but would be in a form that would allow easy integration providing knowledge. At the same time, overwhelming the user with too much information is a problem that should be avoided.

\section{Enhancing Collaboration}

The second area was to provide ways of enhancing collaboration. This involved the ability to bring all the players and data together to more easily solve problems.

Collaboration in aviation operations exists currently in many forms. But it involves low bandwidth, inefficient user interfaces, and slow or spotty turn around times.

\section{Enhancing Existing Interfaces}

The third benefit area we identified was to provide relevant interfaces to the information. This would be to provide interfaces to the user that were easy to use and navigate. We were interested in investigating interfaces that would be easy to integrate or retrofit into existing work areas such as voice control, PDAs, and wireless technology.

\section{Developing a Simulation Platform}

In parallel with this research, we worked to develop a flight simulation environment in which to demonstrate ideas. Given that we had limited time and budget available, we focused on what other researchers were using and COTS (commercial off the shelf) simulators.

Our initial requirements for a flight simulator included having an open programming interface, an out the window view, the ability to retrieve realtime weather data, the ability to portray weather conditions in the out the window view, and the ability to enter and follow a flight plan for a demo scenario.

After reviewing many simulation platforms, we decided upon Microsoft flight simulator 2000 as the basis for our simulator. We enhanced it with $3^{\text {rd }}$ party networking software (the FSUIPC utility developed by Peter Dowson). We also integrated a separate package called Project Magenta, which provides an enhanced MCP (mode control panel), FMS (flight management system), and glass cockpit displays. This combination provided an adequate simulation environment with a realistic out the window view, and also enabled us to write our own programs to extract data, (for example, position or airspeed), from the simulator while flying it. This interface would also allow us to link the simulator with other applications (for example, an Airline Operations Center) via TCP/IP networking. 


\section{Application Focus Areas}

Based on the results of our stakeholder needs analysis, we felt that improvements in cockpit operations, and better interaction with Airline Operations Center (AOC) were application areas where we should focus our develcpment.

We further refined our demonstration applications areas to Cockpit Weather and Traffic, and Cockpit Monitoring and Control. Both included significant interaction with AOC operations.

\section{Cockpit Weather and Traffic}

\section{Motivation}

From our discussions with commercial pilots, we learned that the AOC, which is the airline's ground support team, can often become overloaded and are not able to answer pilot's yueries for information. This can occur when there is significant weather in play or ther: is a problem with another aircraft.

By providing pilots with access to a display of the current weather and the locations of other aircraft similar to that of the AOC. we felt that we could offload the reliance on the AOC and at the same time, could enable the improvement of local and strategic decision making. For example, pilots could determine the best route to Boston after taking off from San Francisco by knowing early on where bad weather systems are located and also by observing where other traffic is going: they could avoid areas which will be greatly impacted with aircraft trying to avoid the same weather systems.

\section{Development}

To provide weather and traffic: for this application we teamed with RLM of Boston, MA. We used RLM's FlightView product, which displays traffic and weather throughout the US and Canada. A user can easily zoom and pan around the display to focus in on an area of interest. An example of the flight view display is shown in Figure 1. In this application, we ran a copy of the Flight View application in both the cockpit and AOC station.
For this application, we reconfigured the Microsoft flight simulator program to suppress the display of cockpit gauges in order to maximize the out the window view. Figure 2 shows a typical display from this application as configured.

We used the FSUIPC and WideFS utilities developed by Peter Dowson to extend the networking capabilities of Microsoft Flight Simulator. These utilities allowed us to interface

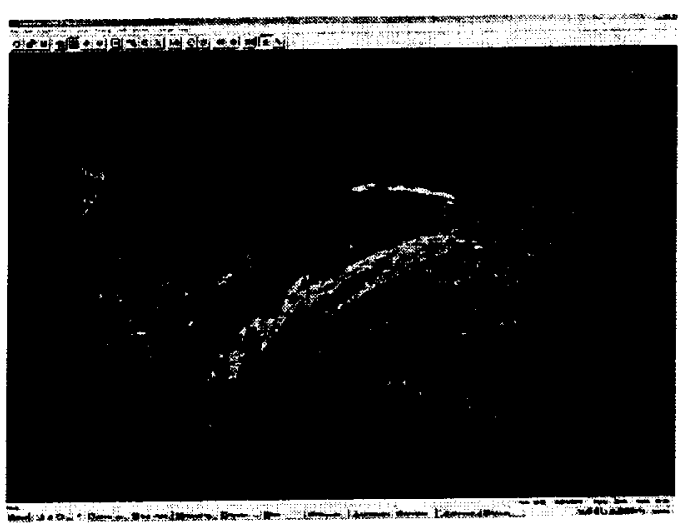

Figure 1. RLM Flight View Weather and Traffic Display.

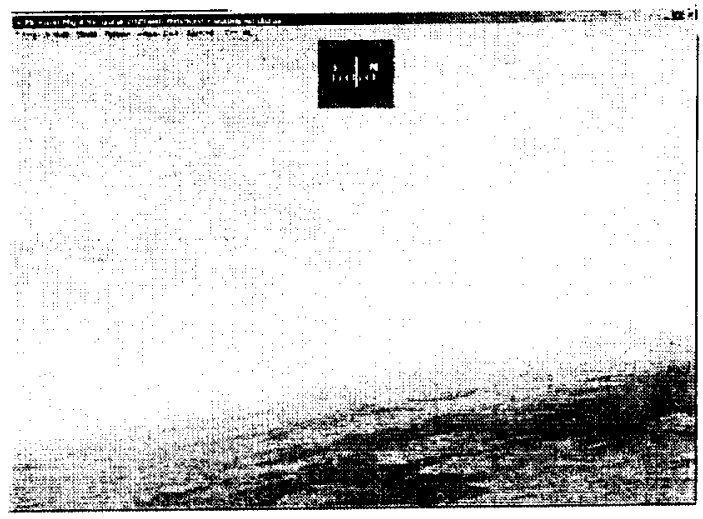

Figure 2. Microsoft Flight Simulator Out the Window Display.

the simulator across an Ethernet network with another PC that represented the AOC station. We used this to simulate the high bandwidth satellite connection between the aircraft and the ground. 
Using this software, a dynanic link library was developed at NASA that allowed applications to read the location, heading and altitude of the flight simulator off of the network. The RLM engineers integrated this software into their FlightView software, which then allowed this program to display a real-time update of the laboratory flight simulator in the live traffic and weather display. Figure 3 shows the resulting architecture of this integrated system.

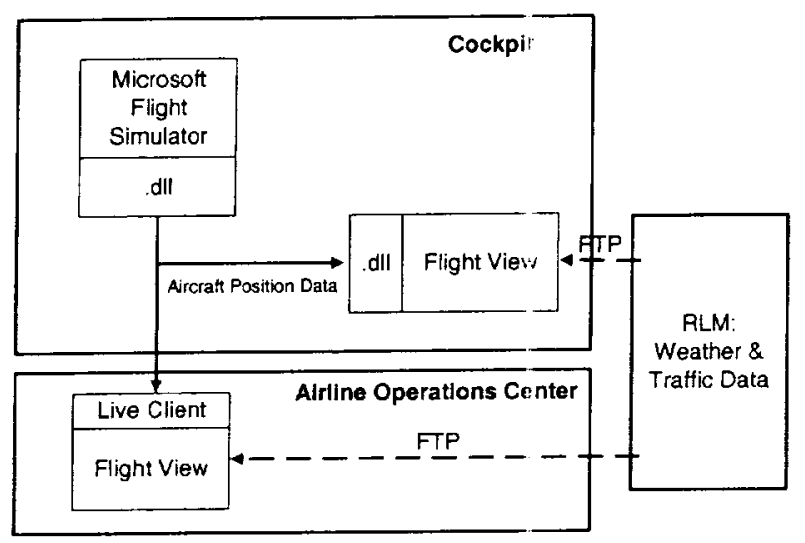

Figure 3. Cockpit Weather and Traffic Application Architecture.

\section{Results}

The bandwidth requirements of this system were minimal at about $3 \mathrm{Kbps}$ uplcaad and less than $1 \mathrm{Kbps}$ download. This rate provided a traffic update every 3 minutes and the weather every 6 minutes, which was the update limit of the Flightview application. At this low bandwidth, this would be a candidate application for multicasting to all aircraft in a fleet over a single transponder.

\section{Directions for Further Development}

We have several ideas for the further development of this application of real time weather and traffic display in the cockpit.

- Integrate additional weather products such as high resolution Doppler radar, upper level winds and other data into the display.

- Verify through human factors studies that the additional data actually improves situational awareness and lower the overall cost of flights, individually or in collaboration with the AOC.

- $\quad$ Provide ways for the AOC to annotate and enhance data presented in the aircraft cockpit (strategic collaboration).

- $\quad$ Model the downloading of weather data from the aircraft to support the development of more accurate models of weather and the atmosphere.

- Investigate new interfaces, such as voice or visual, for the display of data.

\section{AOC Monitoring and Control}

\section{Motivation}

The events of September $11^{\text {th }}$ were a motivating factor in developing an application that would provide an AOC with the ability to monitor the health of an aircraft and crew in flight. We wanted to provide the capability for the AOC to take over and control the aircraft. We also felt that this would be a better test of bandwidth capabilities.

Our motivation to provide cockpit monitoring and control came from reports that detailed what happened during the takeover of the four aircraft. The AOCs sensed that there was a problem but had no way of verifying what the problem was. United Airlines and American Airlines AOCs collaborated and reacted quickly to ground their fleets before any orders from the FAA.

We were interested in collecting multiple streams of data and providing these to the ground. The streams should also complement themselves and allow the discrimination of false indications. We felt that aircraft telemetry (speed, position, heading); video (cockpit and cabin); and pilot health (pulse rate, blood pressure) were the data streams necessary to fully monitor the state of the aircraft and crew.

A further motivation to develop this application is that it would allow us to investigate control station concepts for unmanned aerial vehicles (UAVs) and space assets such as the personal satellite assistant (PSA), space station, and future spacecraft. These systems also have the need 
to provide multiple streams of data (aircraft health, multiple sensors and video data), so the resultant architecture might be a good match.

\section{Development}

We built upon the architecture developed for the Cockpit weather and Traffic Application. We continued to use Flight View on both the cockpit and AOC applications as previously supported by the FSUIPC and WideFS network utilities.

\section{Remote Cockpit Instruments and Control}

We used a plug in to Microsoft Flight Simulator called Project Magenta. developed by Enrico Schiratti. This package provides a networked architecture that allow: multiple cockpit applications, (for example, cockpit instruments, throttles, mode control panel (MCP), and flight management system (FMS)) to send and receive information from the flight simulator. These applications can be co resident or distributed across computers on a network.

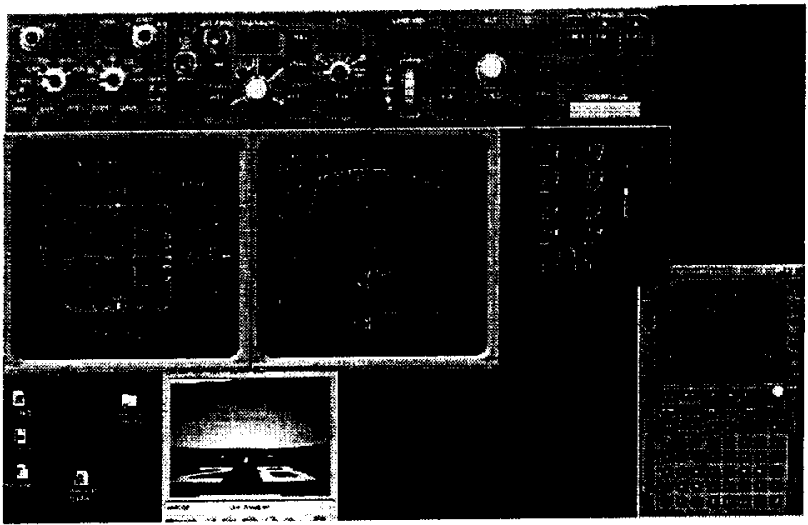

Figure 4. AOC Flight Instrument and Control Display.

Figure 4 shows the flight instrument and control interface that was provided at the AOC station. It consists of a Mode Control Panel, Primary and Navigation displays, Engine Instruments, and a Control Display interface to the Flight Management system. This interface is fully capable of controlling the aircraft simulator remotely.

\section{Cockpit Video Feed}

We next integrated a video camera into the system. We used a USB camera installed on a PC and used a Sorenson video server to transmit the data to the AOC station via the network. This camera was used to provide an out the window view for landing the aircraft. To simulate this video, we positioned the camera so that it was focused on the out the window view display from Microsoft Flight simulator. The output of this at the AOC station can be seen at the bottom of figure 4 .

We also wanted to be able to provide simulated cockpit and cabin views to the AOC that would be selectable. In practice, multiple cameras under a selectable feed could have handled this functionality. For expediency we chose to provide a second video feed of the cabin area using the video provided by the Smart Healthcare Management System described below.

\section{Monitoring Pilot Health}

We wanted to be able to provide a noninvasive monitoring capability of the health (stress or workload) of the pilot. Pulse rate has been found to be a good measure of pilot workload [4] and should be easy to measure. We investigated many systems, including PC based blood pressure monitors, and a pulse rate monitor developed by the Hewlett Packard Palo Alto Research Lab. We eventually found the best solution here at Ames from the Smart Healthcare Management Systems Team, Astrobionics Division.

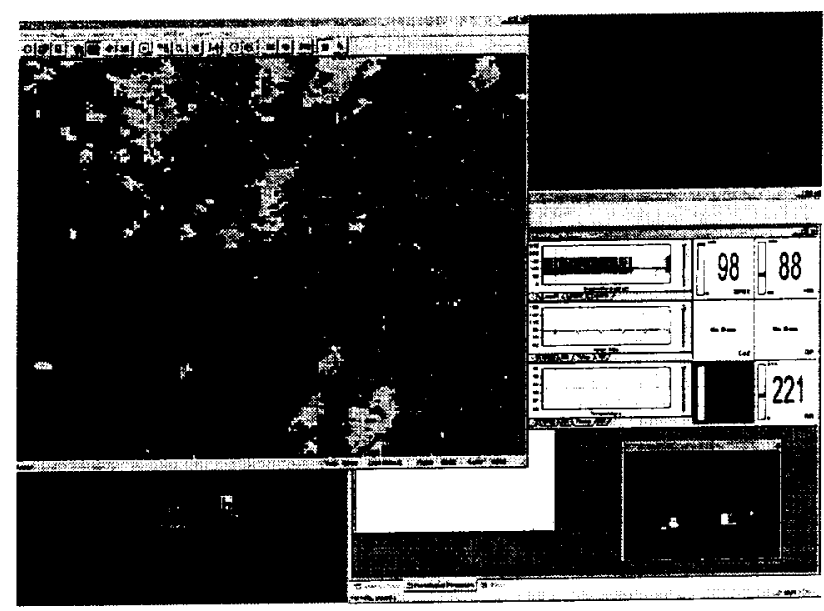

Figure 5. AOC Weather and Pilot Monitoring Displays. 


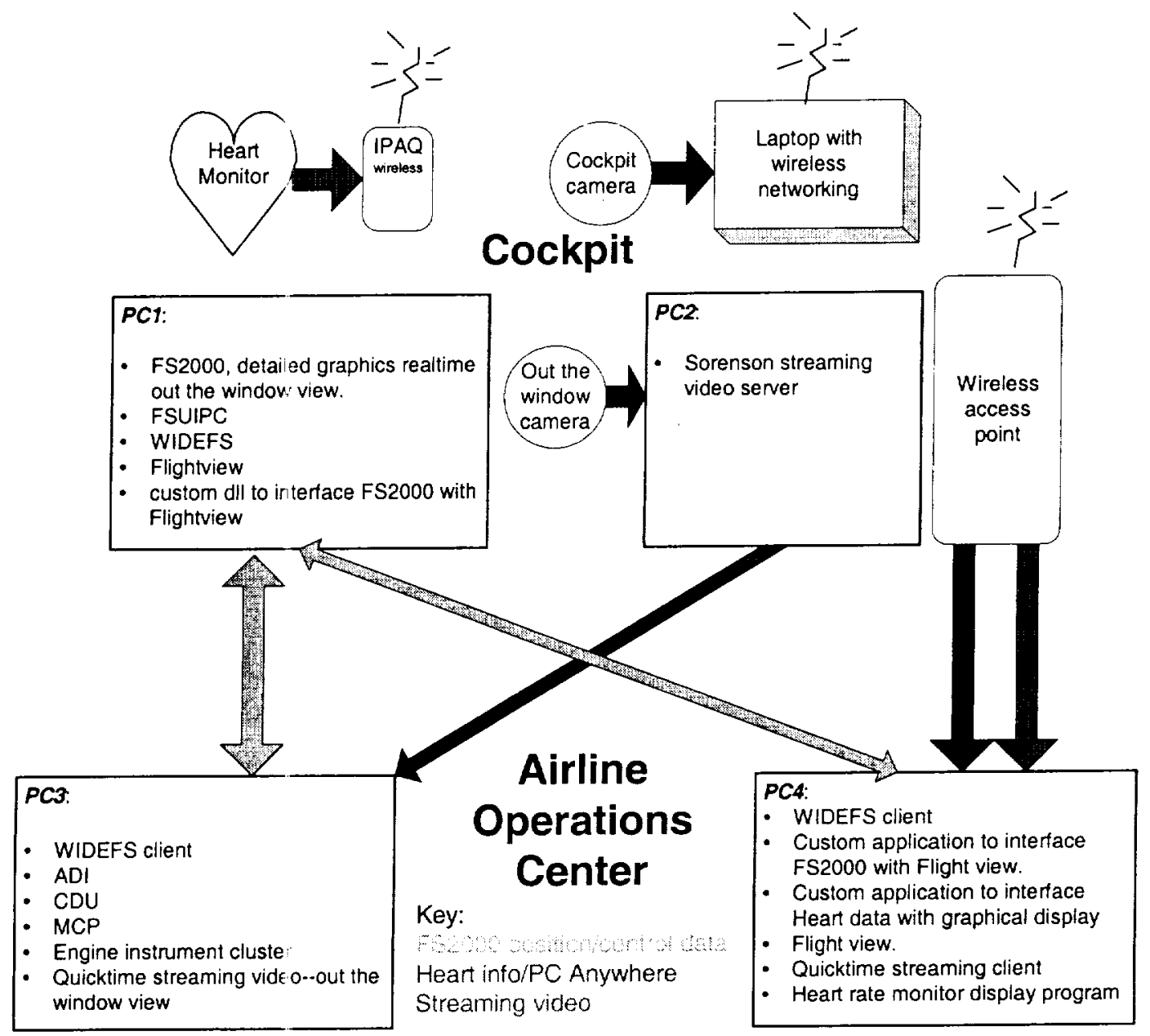

Figure 6. Application Architecture for the Cockpit Monitoring and Control Application.

This system has been under development for several years. Its goal is to provide the capability of remotely monitoring primarily human vital signs via wireless and other networked links. They have developed a specialized monitoring sensor package that can be adhered to a user's body, or could easily be built into a seat. It is also capable of integrating data from any type of telemetry stream, for example engine or fire monitoring.

The system software is portable and is capable of running on most platforms. It was used by our application running on a Compaq IPAQ computer that provided blood pressure, heart rate, and cockpit video over a wireless network to our AOC station. Figure 5 shows the second AOC computer display with the Flight View application in the upper left and the Smart Healthcare Display in the lower right.

\section{Results}

Figure 6 shows the full implementation of the AOC Monitoring and Control simulation including the applications and their distribution among the computers.

Figure 7 shows a diagram that summarizes the bandwidth requirements of the Cockpit Monitoring and Control Application. Recall that this application subsumes the work developed in the Cockpit Weather Application. 


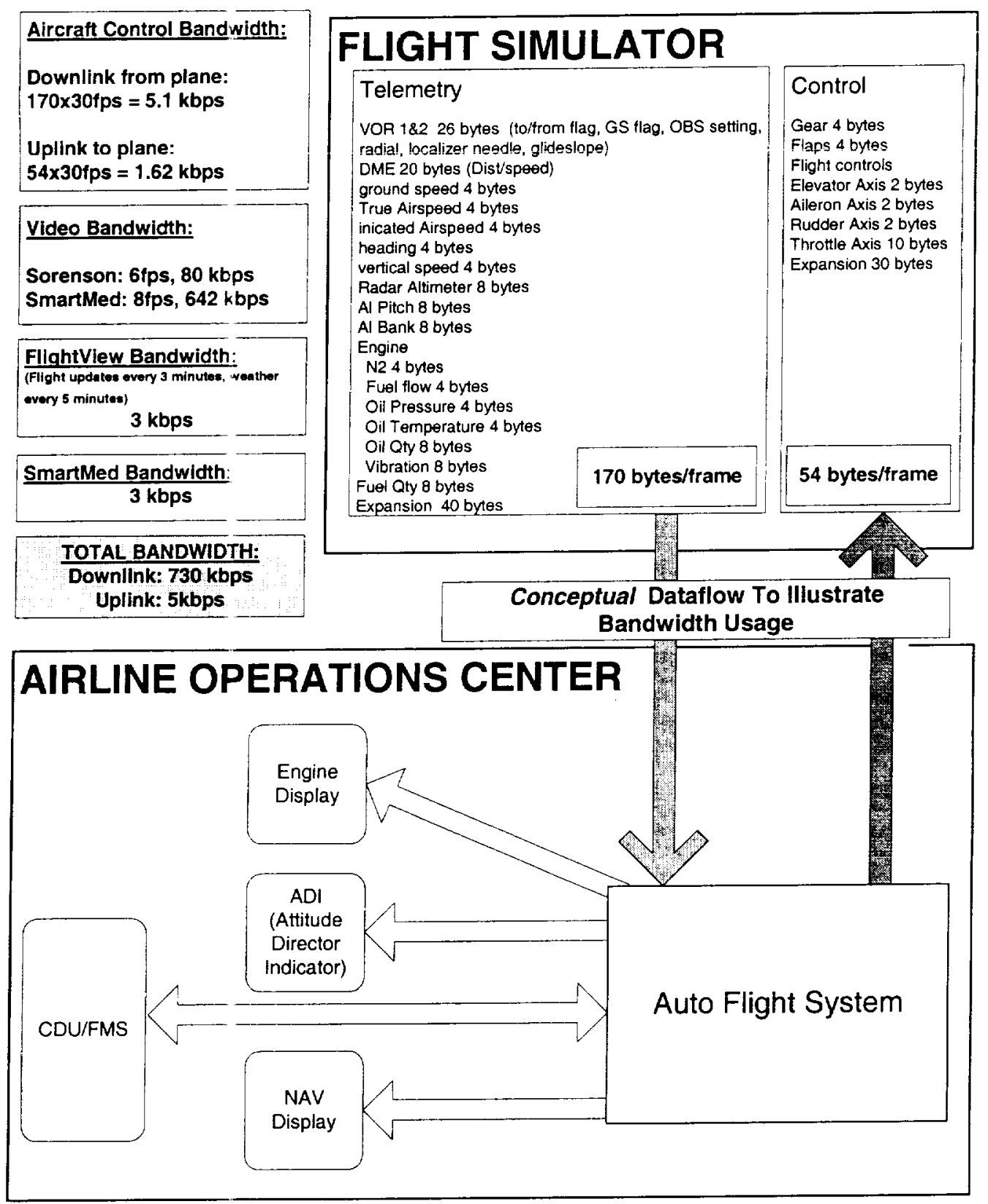

Figure 7. Bandwidth Requirements of the Cockpit Monitoring and Control Application.

This diagram provides a total bandwidth requirement under emergency conditions (all telemetry, video and control links active) of 730 Kbps downlink and 5 Kbps uplink.

Recall that our target values under nominal conditions were $25.6 \mathrm{Kbps}$ downlink and $218 \mathrm{Kbps}$ uplink, and up to $256 \mathrm{Kbps}$ downlirk and $2.18 \mathrm{Mbps}$ uplink available during emergency operations. In this configuration, we far exceeded our limits, but there are several factors that would bring this bandwidth usage into reason.

Because of time and funding limitations we chose to use the video from the Smart Heathcare System without compression for in cockpit video. We feel that if we were to have used Sorenson compression on this data, we could have reduced its 
value from $642 \mathrm{Kbps}$ down to something in the order of $80 \mathrm{Kbps}$. This would have brought our total down to approximately $170 \mathrm{Kbps}$ in an emergency configuration, which would have been well within our limits.

If we were just monitoring the health of the aircraft and pilots, this value would have been within our limits at $8 \mathrm{Kbps}$. Also, we feel that there may be additional opportunities to reduce the bandwidth by adjusting update rates, or actively controlling which streams have priority in different modes (for example, cockpit vide) would have less priority in comparison with out the window video during landing operations).

\section{Further Directions}

In developing this application, we identified several directions in which this system could be expanded to provide additional capabilities.

\section{Smart Monitoring and Alert}

To reduce the amount of dats that would need to be continually downloaded from each aircraft, an on board system could be developed that would actively monitor the aircraft and cabin data streams. It could also be able discern when. for example, extraneous movement in the cabin, a high pulse rate from the pilot, and deviations of course might indicate a possible hijacking or an explosive decompression. The system would notify the AOC and institute a higher bandwidth connection only when a non-nominal situation was thought to exist.

In place, this would allow a much smaller stream of continuous data (indicating that conditions were nominal) to be sent to the AOC, allowing many aircraft to be moni: ored via a single satellite transponder.

\section{Active Streaming}

It is also possible that the datic return capability of the aircraft could improve through the use of alternative technology. This would include both "black-box" information, as well as more advanced data such as streaming video of what is going on in the cockpit. In discussions with fellow researchers after the September $11^{\text {th }}$ incident, there was an interest in showing not only how an incident happened, but also the prior 10 minutes leading up to it.

This data could be continually stored on tape or disk in a black box. However, it was brought to our attention by Robert Pap of the Accurate Automation Corporation that " 15 of the last 16 commercial aircraft that have crashed have included satellite communications equipment." When a problem occurred, this archived data (the last 10 minutes) could be broadcast at some rate over a satellite or aviation specific wireless networks. This data could also be interwoven with real time video data.

\section{Integrated Controls}

The cockpit control interface that we implemented in our AOC simulation was the same interface as that of a commercial aircraft. This interface is quite obvious to commercial pilots but might not be as obvious to a pilot not current on that aircraft or to all AOC personnel. If this interface were implemented it would require a pilot be on staff at all times at that facility. Alternatively, it would require the capability of transferring the control feed to a different facility where a pilot current on that aircraft was available.

To work around this requirement, it should be possible to develop an interface that would allow simpler goal-based text, graphical, or voice commands (for example, fly to this waypoint and hold) to be used. This interface would translate those commands into those that the specific aircraft autopilot under control would understand and follow. This interface would be essential under a mixed fleet situation (for example, an airline with both Boeing and Airbus aircraft) or could also be used to control multiple UAV assets.

\section{Uses of Additional Uplink Data}

In the development of these applications, we were able to fully saturate the channel containing the data coming from the aircraft, but were not able to fully utilize the uplink channel, even though it had much more capability. From what we have learned, this was acceptable, because the limited number of transponders available would force the sharing of this resource through the multicast of data to many aircraft. 
However, additional research is necessary to investigate what applications to pilots and other aviation stakeholders could use more of this channel under emergency conditions. This would include both the applications and the phase of flight in which they would be used.

\section{Summary}

In the BEFO project, we researched and identified applications of high bandwidth data in commercial aviation. We also worked to gain a better understanding of the availatle bandwidth and how it could be applied in this environment. In the process, we identified and worked with several teaming partners. We were able to develop a highbandwidth simulation architecture and demonstrated it on two sample applications. We identified several areas where these applications could be expanded to support additional functionality. We are interested in expanding this work to investigate and evaluate technologies that can be applied to commercial flight, UAV and space applications.

\section{Acknowledgements}

We would like to thank Dr. Mike Lowry and Dr. David Maluf, Computational Systems Branch, NASA Ames Research Center for initiating and supporting this effort.

We would also like to thank rnembers of the Smart Healthcare Management Sy stems Project team that contributed to the success of our demonstration: John Hines, Carsten Mundt, Kevin Montgomery, and Elizabeth Roland.

We'd also like to thank Mike Santomauro of the Oakland Flight Service Station for access to that facility.

\section{References}

[1] Brooks, David E., Gregory W. Romaniak, Brian D. Frantz, 2000, AeroSAPIENT Final Report, DC 8 Flight Experiments, Cleveland, Ohio, NASA Glenn Research Center.

[2] Dorr, Les, 2000, Controller Pilot Data Link System (CPDLC), FAA Fact Sheet, Washington, DC, Federal Aviation Administration.

[3] Capstone Program Management Office, 2001, Capstone Program Plan, Anchorage, AK, Federal Aviation Administration.

[4] Brenner, Malcolm, E. Thomas Doherty, and Thomas Shipp, 1984, Speech measures indicating workload demand, Alexandria, VA, Aviation, Space, and Environmental Medicine, 56:21-6. 